\title{
Pediatric Rheumatology - a continuation of Pediatric Rheumatology Online Journal
}

\author{
Alberto Martini*1 and Charles H Spencer ${ }^{2}$
}

\author{
Address: ${ }^{1}$ Professor Of Pediatrics, Ospedale Gaslini, Genova, Italy and ${ }^{2}$ Professor of Pediatrics, University of Chicago, Chicago, Illinois, USA \\ Email: Alberto Martini* - albertomartini@ospedale-gaslini.ge.it; Charles H Spencer - cspencer@larabida.org \\ * Corresponding author
}

Published: 2 April 2007

Pediatric Rheumatology 2007, 5:I doi:10.1 I86/1546-0096-5-I
Received: 2 April 2007

Accepted: 2 April 2007

This article is available from: http://www.ped-rheum.com/content/5/I/I

(C) 2007 Martini and Spencer; licensee BioMed Central Ltd.

This is an Open Access article distributed under the terms of the Creative Commons Attribution License (http://creativecommons.org/licenses/by/2.0), which permits unrestricted use, distribution, and reproduction in any medium, provided the original work is properly cited.

We welcome you to Pediatric Rheumatology, now published by BioMed Central. Pediatric Rheumatology is a continuation of the open access Pediatric Rheumatology Online Journal, launched in 2003 as the first solely pediatric rheumatology journal.

Our mission is to further research, scholarship, and education in pediatric rheumatology throughout the world and thereby improve the care of children with arthritis and rheumatic diseases. We hope to speed the growth of pediatric rheumatology in every country. We believe that a dedicated pediatric rheumatology journal has a better opportunity to help develop our field than existing, predominantly adult rheumatology journals. The journal is independent and international and is purposefully free of direct association with any one institution, organization, or commercial enterprise other than BioMed Central.

Our choice of the open access venue is intentional. We wish the pediatric rheumatology information in this journal to be freely available to anyone. We believe that this model is optimal for growth of pediatric rheumatology in both developed and developing countries [1,2]. Why is the online, open access venue preferable to the traditional subscription journals in rheumatology? First, all articles become freely and universally accessible online and so an author's work can be read by anyone at no cost. Articles, photos, figures, tables, and other components can be freely downloaded. Second, the authors, not the publishers, hold the copyright for their work and grant anyone the right to reproduce and disseminate the article, as long it is correctly cited and no errors introduced. Third, Pediatric Rheumatology's articles will be archived in PubMed
Central, the US National Library of Medicine's full-text repository of life science literature. These articles will also be archived in the repositories at the University of Potsdam in Germany, at INIST in France, the e-Depot, the National Library of the Netherlands' digital archive of all electronic publications, and in the new UK PubMed.

Why should we join the shift towards online, open access publishing? The traditional business model for scientific publishers requires restriction of access to published research so that the publishers can recoup the costs of publication. This restriction has deleterious effects, limiting full use of digital technology, and can be considered to be contrary to the interests of authors, funders, and the scientific and medical community as a whole. This traditional system is straining as increasing amounts of research are being published while library budgets have remained static. The open access approach treats publication as the last step in the research process:

1) The article processing charge (APC) covers the cost of publication so that there is free and immediate access to research and education articles.

2) The APCs ensure transparency and allow publishers to compete to provide the best service at the best price.

3) By linking the cost of publication to research budgets, APCs allow the publishing system to adjust to the everincreasing volume of clinical and basic research [3].

Pediatric Rheumatology is open to submissions on all aspects of pediatric rheumatology, including: editorials; 
commentaries; research, both clinical and basic; clinical and basic science reviews; rheumatologic reviews for the generalist; significant case series or case reports; book reviews; letters to the editors; and drug studies. Submissions are online, reviews and decisions occur within four weeks in most cases, and publication online is rapid and continuous.

We hope that Pediatric Rheumatology will be useful to pediatricians, pediatric rheumatologists, adult rheumatologists, other professionals, and the lay public. Let's work together to help our kids with rheumatic diseases.

\section{Competing interests}

Alberto Martini, MD and Charles H. Spencer, MD are CoEditor-in-Chiefs of Pediatric Rheumatology.

\section{References}

I. Suber P: Open access, impact, and demand: Why some authors self archive their articles. BMJ 330(7500): 1097-1098. 2005 May 14

2. Tan-Torres Edejer T: Disseminating Health information in developing countries: The role of the internet. BMJ 2000, 32 I:797-800.

3. Frequently asked questions about BioMed Central's article processing charges [http://www.biomedcentral.com/info/about/ apcfaq]
Publish with Biomed Central and every scientist can read your work free of charge

"BioMed Central will be the most significant development for disseminating the results of biomedical research in our lifetime. " Sir Paul Nurse, Cancer Research UK

Your research papers will be:

- available free of charge to the entire biomedical community

- peer reviewed and published immediately upon acceptance

- cited in PubMed and archived on PubMed Central

- yours - you keep the copyright

Submit your manuscript here:

http://www.biomedcentral.com/info/publishing_adv.asp 